\title{
Free Energies and Minimal States for Scalar Linear Viscoelasticity
}

John Murrough Golden

Technological University Dublin, Murrough.Golden@TUDublin.ie

Follow this and additional works at: https://arrow.tudublin.ie/scschmatart

Part of the Mathematics Commons

\section{Recommended Citation}

Golden, M. (2015) Free Energies and Minimal States for Scalar Linear Viscoelasticity. Journal of elasticity, published online Oct. 14th. 2015. Springer. DOI10.1007/s10659-015-9549-y

This Article is brought to you for free and open access by the School of Mathematics at ARROW@TU Dublin. It has been accepted for inclusion in Articles by an authorized administrator of ARROW@TU Dublin. For more information, please contact arrow.admin@tudublin.ie, aisling.coyne@tudublin.ie,gerard.connolly@tudublin.ie.

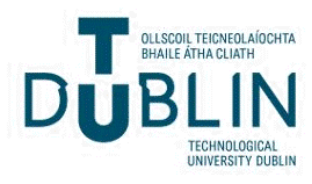




\title{
Free Energies and Minimal States for Scalar Linear Viscoelasticity
}

\author{
Giovambattista Amendola $^{1}$. Mauro Fabrizio ${ }^{2}$. \\ John Murrough Golden ${ }^{3}$
}

Received: 26 February 2015

(C) Springer Science+Business Media Dordrecht

\begin{abstract}
The concept of a minimal state was introduced in recent decades, based on earlier work by Noll. The property that a given quantity is a functional of the minimal state is of central interest in the present work. Using a standard representation of a free energy associated with a linear memory constitutive relation, a new condition, involving linear functionals, is derived which, if satisfied, ensures that the free energy is a functional of the minimal state. Using this result and recent work on constructing free energy functionals, it is shown that if the kernel of the rate of dissipation functional is given by sums of products, the associated free energy functional is a functional of the minimal state.
\end{abstract}

Keywords Viscoelasticity · Thermodynamics · Memory effects · Free energy

Mathematics Subject Classification (2010) 74D05 · 80A17

\section{Introduction}

There are generally many free energies associated with a material with memory. They form a bounded convex set with a minimum and a maximum element ([14], for example), which we denote by $\mathcal{F}$.

J.M. Golden

murrough.golden@dit.ie

G. Amendola

amendola@dma.unipi.it

M. Fabrizio

fabrizio@dm.unibo.it

1 Dipartimento di Matematica, ???, Largo Bruno Pontecorvo 5, 56127 Pisa, Italy

2 Dipartimento di Matematica, ???, Piazza di Porta S. Donato 5, 40127 Bologna, Italy

3 School of Mathematical Sciences, Dublin Institute of Technology, Kevin Street, Dublin 8, Ireland 
Explicit algebraic representations of the minimum and related free energies have been given for materials with memory which have constitutive equations for stress $^{1}$ given by linear functionals of the strain; see for example [1, 2, 4, 10, 12, 15-21].

More classical examples of free energies were constructed and considered in earlier references, namely the Graffi-Volterra and Dill functionals discussed in $[2,7,8,14,24,26]$ and other papers. These are denoted by $\psi_{G V}(t)$ and $\psi_{\text {Dill }}(t)$ respectively. There is also a free energy functional $\psi_{F}(t)$ which was introduced more recently [11] and is an explicit functional of the minimal state (henceforth abbreviated to FMS).

These are all quadratic functionals which yield linear constitutive equations for the stress, in a mechanics context. They are easily generalized to materials with linear memory constitutive relations but where the memory-independent contribution may be non-linear. A generalization of such quadratic functionals, yielding non-linear constitutive relations has been proposed recently [22].

It should be noted that some functionals are free energies only for certain types of material, so that the size of $\mathcal{F}$ will depend on material properties. Examples of this are $\psi_{G V}(t)$, $\psi_{\text {Dill }}(t)$ and $\psi_{F}(t)$. Those with the least constraints on the material are the minimum free energy and also the family of related functionals discussed in for example $[2,18]$ (though this family is non-trivial only for relaxation functions with no branch cut singularities in the frequency domain).

Recent work [20] seeks to systematically explore existing and new categories of free energy functionals in $\mathcal{F}$, using a novel technique. Free energies that are quadratic functionals of quantities known to be FMSs are explored in another recent paper [21]. It is found that this is a very restricted category.

The concept of a minimal state was introduced in recent decades ([2, 7, 8, 10, 15, 25], for example) based on earlier work by Noll [27]. Recent work on this topic in the context of fractional hereditary materials may be found in [9, 13]. The term minimal state was first used in [15]. The property that a given quantity is a FMS is of central interest in the present work. Most but not all of the free energies discussed above are FMSs. This is a property that involves conditions on quadratic functionals. In the present work, we present an alternative linear functional condition, which holds if and only if the free energy of interest is a FMS. Because it is a linear rather than a quadratic, it is easier to explore and to apply in new contexts.

The use of this new formula is illustrated by applying it to test whether certain standard free energies are FMSs. This involves confirming results already known by alternative arguments. However, it is also applied to the new free energy functionals introduced in [20]. Indeed, it is shown that if the kernel of the rate of dissipation functional is given by sums of products, the associated free energy functional is a FMS.

Remark 1 Such categories of free energies are very general and this suggests a conjecture that the only free energies given by quadratic functionals of the kind discussed in this work

\footnotetext{
${ }^{1}$ We consider for definiteness here isothermal mechanical problems, indeed those for solid viscoelastic materials. Also, only the scalar case is considered, which simplifies the algebra and allows us to focus on the essential structure of the arguments. It must be emphasized however that similar results have been given, with little extra difficulty, for viscoelastic fluids, non-isothermal problems, electromagnetic and non-simple materials with memory, and also for the general tensor theories relating to all of these. Specifically, the fundamental result given by Proposition 1 in Sect. 4 can be shown to hold, with some rephrasing of the argument, for the full tensor case. However, the developments of Sects. 9 and 10 generalize similarly only subject to factorization restrictions, which were first discussed for the simplest case in [10] (see also [2], p. 256).
} 
which are not FMSs are degenerate cases, in particular the Graffi-Volterra functional and the work function, both discussed in Sect. 4.1, and any combination of free energies including these.

Free energy functionals are useful in defining the topology of the space of states, when studying stability and related problems for the integro-differential equations describing the evolution of materials with memory, subject to stress. A new approach (for example, [2], p. 390) to such problems has been initiated by Fabrizio recently, which has a number of advantages and which seeks to express function spaces in terms of minimal states and use free energies which are FMSs. Another use for free energies is in modeling dissipation of energy in materials with memory. This requires choosing a functional that approximates, in some fashion, the physical free energy $[18,23]$. If some property related to this phenomenon has been established for a given class of histories, then this class may be considerably extended if the material has the property that minimal states are non-singleton and the free energy is a FMS.

Regarding the notational convention for referring to equations, we adopt the following rule. A group of relations with a single equation number $(* * *)$ will be individually labeled by counting "=" signs or " $<$ ", " $>$ ", " $\geq$ " and " $\leq$ ". Thus, $(* * *)_{5}$ refers to the fifth "=" sign, if all the relations are equalities. Relations with " $\in$ " are ignored for this purpose.

\section{Models of Materials with Memory}

For simplicity, we confine the discussion to the scalar theory. The strain at time $t$ and its history are $E(t)$ and $E^{t}$ respectively, where

$$
E^{t}(s)=E(t-s), \quad s \in \mathbb{R}^{+},
$$

while the relative history is given by

$$
E_{r}^{t}(s)=E^{t}(s)-E(t), \quad s \in \mathbb{R}^{+} .
$$

For simplicity, we take the history to be continuous, in particular at $s=0$. The stress is denoted by $T(t)$. The general form of the constitutive relation is

$$
T(t)=\widetilde{T}\left(E^{t}, E(t)\right)
$$

where it is understood that $\widetilde{T}$ is a functional of $E^{t}$ and a function of $E(t)$. The general form of a free energy functional is denoted by

$$
\psi(t)=\tilde{\psi}\left(E^{t}, E(t)\right) \geq 0,
$$

where, as with $\widetilde{T}$, the quantity $\tilde{\psi}$ is a functional of $E^{t}$ and a function of $E(t)$. Let $E^{\dagger}$ be the static history, equal to $E(t)$ at the current and all past times. Then

$$
\tilde{\psi}\left(E^{\dagger}, E(t)\right)=\tilde{\phi}(E(t))=\phi(t) \geq 0,
$$


where $\widetilde{\phi}(E(t))$ is the equilibrium free energy. This is a definition of $\widetilde{\phi}$, which must have the same form for any choice of free energy. The abbreviation to $\phi(t)$ will be frequently used.

\subsection{Required Properties of a Free Energy}

We denote a particular free energy at time $t$ by $\psi(t)=\tilde{\psi}\left(E^{t}, E(t)\right)$. Certain properties of free energies, derived in [5], have been used in [10, 14, 17] and elsewhere, to characterize such functionals. These are now listed.

P1. We have

$$
\frac{\partial \tilde{\psi}\left(E^{t}, E(t)\right)}{\partial E(t)}=\frac{\partial \psi(t)}{\partial E(t)}=T(t)=\widetilde{T}\left(E^{t}, E(t)\right) .
$$

Thus, any choice of free energy must yield the same $\widetilde{T}$, according to this prescription. $\mathrm{P} 2$. For any history and current value $\left(E^{t}, E(t)\right)$,

$$
\tilde{\psi}\left(E^{t}, E(t)\right) \geq \tilde{\phi}(E(t)),
$$

where equality is achieved for the static history $E^{\dagger}$.

P3. We have

$$
\dot{\psi}(t)+D(t)=T(t) \dot{E}(t), \quad D(t) \geq 0,
$$

where $D(t)$ is the rate of energy dissipation associated with $\psi(t)$. The first relation is a statement of the first law, while the non-negativity of $D(t)$ is in effect the second law.

These are a version of the Graffi conditions for a free energy. Properties P1 and P2 follow from the second law, which is included in P3 [5, 22].

The work function is given by

$$
W(t)=\int_{-\infty}^{t} T(u) \dot{E}(u) d u=\widetilde{W}\left(E^{t}, E(t)\right),
$$

where it is assumed that the integral exists.

The quantity $W(t)$ behaves similarly to a free energy functional with zero dissipation rate associated with it (for example, [2, 22]). In particular, it follows from (2.9) that

$$
\dot{W}(t)=T(t) \dot{E}(t),
$$

which is (2.8) or P3 for $D(t)=0$.

를 Springer 


\section{General Relationships}

The constitutive relations with linear memory terms have the equivalent forms

$$
\begin{aligned}
T(t) & =T_{e}(t)+\int_{0}^{\infty} G^{\prime}(u) E_{r}^{t}(u) d u \\
& =T_{0}(t)+\int_{0}^{\infty} G^{\prime}(u) E^{t}(u) d u \\
& =T_{e}(t)+\int_{0}^{\infty} \widetilde{G}(u) \dot{E}^{t}(u) d u, \\
T_{0}(t) & =T_{e}(t)+\left(G_{0}-G_{\infty}\right) E(t), \\
G^{\prime}(u) & =\frac{d}{d u} G(u), \quad \widetilde{G}(u)=G(u)-G_{\infty}, \\
\dot{E}^{t}(u) & =\frac{\partial}{\partial t} E^{t}(u)=-\frac{\partial}{\partial u} E^{t}(u)=-\frac{\partial}{\partial u} E_{r}^{t}(u), \quad \ddot{E}^{t}(u)=-\frac{\partial}{\partial u} \dot{E}^{t}(u),
\end{aligned}
$$

where $T_{e}(t)$ is the stress function for the equilibrium limit $\left(E_{r}^{t}(u)=0, u \in \mathbb{R}^{+}\right)$and the quantity $G(\cdot): \mathbb{R}^{+} \mapsto \mathbb{R}^{+}$is the relaxation function of the material. We define

$$
G_{\infty}=G(\infty), \quad G_{0}=G(0), \quad \widetilde{G}(0)=G_{0}-G_{\infty}=\widetilde{G}_{0}
$$

The assumption is made that

$$
\widetilde{G}, G^{\prime} \in L^{1}\left(\mathbb{R}^{+}\right) \cap L^{2}\left(\mathbb{R}^{+}\right),
$$

which will be relevant in the context of taking the Fourier transform of these quantities. The quantity $T_{e}(t)$ in (3.1) is given by

$$
T_{e}(t)=\frac{\partial \phi(t)}{\partial E(t)}
$$

which is the equilibrium limit of (2.6). For a completely linear material,

$$
\tilde{\phi}(E(t))=\phi_{l}(t)=\frac{1}{2} G_{\infty} E^{2}(t)
$$

and $T_{0}(t), T_{e}(t)$ are given by the form

$$
T_{0}(t)=G_{0} E(t), \quad T_{e}(t)=G_{\infty} E(t)
$$




\subsection{Representation of a Free Energy as a Quadratic Functional}

For a scalar theory with a linear memory constitutive relation for the stress, the most general form of a free energy is

$$
\begin{aligned}
\psi(t) & =\phi(t)+\frac{1}{2} \int_{0}^{\infty} \int_{0}^{\infty} E_{r}^{t}(s) \mathcal{G}(s, u) E_{r}^{t}(u) d s d u \\
& =S(t)+\frac{1}{2} \int_{0}^{\infty} \int_{0}^{\infty} E^{t}(s) \mathcal{G}(s, u) E^{t}(u) d s d u \\
& =\phi(t)+\frac{1}{2} \int_{0}^{\infty} \int_{0}^{\infty} \dot{E}^{t}(s) \widetilde{G}(s, u) \dot{E}^{t}(u) d s d u \\
\mathcal{G}(s, u) & =\frac{\partial^{2}}{\partial s \partial u} G(s, u)=G_{12}(s, u), \quad \widetilde{G}(s, u)=G(s, u)-G_{\infty},
\end{aligned}
$$

where the subscripts in (3.7) $)_{5}$ and below indicate differentiation with respect to the first and second argument, while $S(t)$ has the form

$$
S(t)=\phi(t)+\left(T(t)-T_{0}(t)\right) E(t)+\frac{1}{2}\left(G_{0}-G_{\infty}\right) E^{2}(t) .
$$

The quantity $T_{0}(t)$ is defined by $(3.1)_{4}$. There is no loss of generality in taking

$$
\widetilde{G}(s, u)=\widetilde{G}(u, s), \quad \mathcal{G}(s, u)=\mathcal{G}(u, s) .
$$

The following properties of $G$ are assumed to hold for $s, u \in \mathbb{R}^{+}$:

$$
\begin{gathered}
G(\infty, u)=G(s, \infty)=G_{\infty}, \\
G_{1}(s, \infty)=G_{2}(\infty, u)=0, \quad G_{1}(\infty, s)=G_{2}(u, \infty)=0 .
\end{gathered}
$$

The relaxation function $G(u)$ is given by

$$
G(u)=G(0, u)=G(u, 0), \quad \forall u \in \mathbb{R}^{+},
$$

yielding

$$
G^{\prime}(u)=G_{2}(0, u)=G_{1}(u, 0) .
$$

Relation (3.12) ensures that P1 or (2.6), applied to (3.7) 1 , yields (3.1). Properties (3.10) and (3.12) are required in the derivation of $(3.7)_{2}$ from $(3.7)_{1}$.

The Graffi condition P2, given by (2.7), requires that the kernels $\mathcal{G}$ and $\widetilde{G}$ must be such that the integral terms in $(3.7)_{1,3}$ are non-negative.

Note that the quantity $G(u)$ is the same for all choices of free energy, i.e. for all choices of $G(s, u)$, since it generates the defining constitutive equation for the material. This is a constraint on $G(s, u)$.

Referring to the quantity $S(t)$, given by (3.8) and used in $(3.7)_{2}$, we see that

$$
\frac{\partial}{\partial E(t)} S(t)=T(t)
$$

which is $\mathrm{P} 1$ or $(2.6)$ for the form $(3.7)_{2}$.

Springer 
The rate of dissipation can be deduced from (2.8), (3.7) and (3.1) $8,9,10$ to be

$$
\begin{aligned}
D(t) & =-\frac{1}{2} \int_{0}^{\infty} \int_{0}^{\infty} \dot{E}^{t}(s) K(s, u) \dot{E}^{t}(u) d s d u \\
& =-\frac{1}{2} \int_{0}^{\infty} \int_{0}^{\infty} E_{r}^{t}(s) \mathcal{K}(s, u) E_{r}^{t}(u) d s d u,
\end{aligned}
$$

where

$$
K(s, u)=G_{1}(s, u)+G_{2}(s, u), \quad \mathcal{K}(s, u)=\mathcal{G}_{1}(s, u)+\mathcal{G}_{2}(s, u)=K_{12}(s, u) .
$$

The quantity $G$ must be such that the integrals in (3.14) are non-positive, as required by P3 of the Graffi conditions. The quantities $K$ and $\mathcal{K}$ can also be taken to be symmetric in their arguments, as in (3.9).

There are two equivalent alternatives for the developments outlined in the present work, the first being to use $\widetilde{G}(s, u), K(s, u), \dot{E}^{t}(s)$ and the second to use $\mathcal{G}(s, u), \mathcal{K}(s, u), E_{r}^{t}(s)$. We will have occasion to use both notations, though mainly the first.

\subsection{The Work Function}

This quantity, given by $(2.9)_{1}$, can be put in the following forms ([2], p. 153 and earlier references cited therein):

$$
\begin{aligned}
W(t) & =\phi(t)+\frac{1}{2} \int_{0}^{\infty} \int_{0}^{\infty} \dot{E}^{t}(s) \widetilde{G}(|s-u|) \dot{E}^{t}(u) d u d s \\
& =\phi(t)+\frac{1}{2} \int_{0}^{\infty} \int_{0}^{\infty} E_{r}^{t}(s) \frac{\partial^{2}}{\partial s \partial u} G(|s-u|) E_{r}^{t}(u) d u d s .
\end{aligned}
$$

We see that it can be cast in the forms $(3.7)_{1,3}$ by putting

$$
\widetilde{G}(s, u)=\widetilde{G}(|s-u|), \quad G_{12}(s, u)=\frac{\partial^{2}}{\partial s \partial u} G(|s-u|) .
$$

From the integrated form of (2.8), we have in general,

$$
\psi(t) \leq W(t) .
$$

\section{Minimal States}

This topic will be dealt with here in an elementary manner. For more formal treatments, using states and processes, we refer to [2] and earlier work cited in that reference.

A viscoelastic state is defined in general by the history and current value of strain $\left(E^{t}, E(t)\right)$. The concept of a minimal state can be expressed as follows: two viscoelastic states $\left(E_{1}^{t}, E_{1}(t)\right),\left(E_{2}^{t}, E_{2}(t)\right)$ are equivalent or in the same equivalence class or minimal state if for

$$
E_{1}(t+u)=E_{2}(t+u), \quad \forall u \geq 0,
$$

we have

$$
T_{1}(t+u)=T_{2}(t+u), \quad \forall u \geq 0 .
$$


It is of interest to express these conditions for linear materials. Let us define

$$
I^{t}(\tau)=\int_{0}^{\infty} G^{\prime}(s+\tau) E_{r}^{t}(s) d s=\int_{0}^{\infty} \widetilde{G}(s+\tau) \dot{E}^{t}(s) d s,
$$

for any history and

$$
\begin{aligned}
& I_{i}^{t}(\tau)=\int_{0}^{\infty} G^{\prime}(s+\tau) E_{i r}^{t}(s) d s=\int_{0}^{\infty} \widetilde{G}(s+\tau) \dot{E}_{i}^{t}(s) d s, \quad i=1,2, \\
& I_{d}^{t}(\tau)=\int_{0}^{\infty} G^{\prime}(s+\tau) E_{d r}^{t}(s) d s=\int_{0}^{\infty} \widetilde{G}(s+\tau) \dot{E}_{d}^{t}(s) d s
\end{aligned}
$$

for the histories $E_{1}^{t}$ and $E_{2}^{t}$, where

$$
E_{d}^{t}(s)=E_{2}^{t}(s)-E_{1}^{t}(s), \quad \forall s \geq 0, \quad E_{d}(t)=E_{2}(t)-E_{1}(t) .
$$

The second relation is of course a special case of the first. The condition that $E_{1}^{t}$ and $E_{2}^{t}$ are equivalent histories then takes the form

$$
E_{d}(t)=0, \quad I_{d}^{t}(\tau)=I_{2}^{t}(\tau)-I_{1}^{t}(\tau)=0, \quad \forall \tau \geq 0 .
$$

Note that (4.6) is also the condition that $E_{d}^{t}$ is equivalent to the zero history.

Remark 2 A functional of $\left(E^{t}, E(t)\right)$ which yields the same value for all members of the same minimal state is referred to as a FMS or a minimal state variable. In particular, the quantity $I^{t}$, defined by (4.3), is a FMS.

A fundamental distinction between materials is that for certain relaxation functions, namely those with only isolated singularities (in the frequency domain), the set of minimal states is non-singleton, while if some branch cuts are present in the relaxation function, the material has only singleton minimal states ([2], p. 342). For the singleton case, the only histories satisfying (4.6) are the trivial choices yielding $E_{d}^{t}(s)=0, \forall s \geq 0$.

The results we seek to show are also trivial in this case. The interesting situation is where (4.6) applies for $E_{d}^{t}(s) \neq 0$, which corresponds to non-singleton minimal states. It will therefore be assumed that the relaxation function of the material under consideration has only isolated singularities.

For such materials, there is a maximum free energy that is less than the work function $W(t)$ and also a range of related intermediate free energies. Furthermore, the free energy functional is positive semi-definite ([2], p. 152).

The simplest case of isolated singularities occurs in discrete spectrum materials (see Sect. 6).

A free energy $\psi(t)=\tilde{\psi}\left(E^{t}, E(t)\right)$, is a FMS if it has the property that any two members $\left(E_{1}^{t}, E(t)\right),\left(E_{2}^{t}, E(t)\right)$ of the same minimal state yield equal values of $\tilde{\psi}$, or

$$
\tilde{\psi}\left(E_{1}^{t}, E(t)\right)=\tilde{\psi}\left(E_{2}^{t}, E(t)\right) .
$$

A free energy need not have this property, though most of those which are of interest are in fact FMSs.

We are using a pragmatic definition of a free energy, namely a functional that obeys the Graffi conditions listed in Sect. 2.1. Other definitions exist, in particular the more abstract 
one discussed in [7, 8], with reference to the axiomatic formulation of Coleman and Owen [6]. Some connections between these two definitions are considered in [7, 8] and in for example [10]. For the more abstract formulation, all free energy functionals will be FMSs.

Remark 3 In the discussion (4.1)-(4.6), the history $E_{1}^{t}$ can be chosen arbitrarily, while $E_{2}^{t}$ is restricted to a degree by the conditions (4.6). This observation is important for Proposition 1 below.

Relations (4.1) and (4.2) for $u=0$ become

$$
E_{1}(t)=E_{2}(t), \quad T_{1}(t)=T_{2}(t),
$$

or

$$
E_{d}(t)=0, \quad I_{d}^{t}(0)=\int_{0}^{\infty} G^{\prime}(s) E_{d}^{t}(s) d s=0 .
$$

Observe that they yield the equality

$$
S_{1}(t)=S_{2}(t),
$$

where $S_{1}$ and $S_{2}$ are the function $S$, defined by (3.8), for histories and current values $\left(E_{1}^{t}, E_{1}(t)\right)$ and $\left(E_{2}^{t}, E_{2}(t)\right)$, respectively.

Relation (4.7) is a constraint on quadratic functionals of the history. We now derive a linear condition that is equivalent to (4.7).

Proposition 1 If (4.9) holds for $E_{d}^{t}$, defined by (4.5) $)_{1}$, then the condition (4.7) is equivalent to either

$$
\begin{aligned}
& \int_{0}^{\infty} \mathcal{G}(s, u) E_{d}^{t}(s) d s=\int_{0}^{\infty} \mathcal{G}(u, s) E_{d}^{t}(s) d s=0, \quad \text { or } \\
& \int_{0}^{\infty} \widetilde{G}(s, u) \dot{E}_{d}^{t}(s) d s=\int_{0}^{\infty} \widetilde{G}(u, s) \dot{E}_{d}^{t}(s) d s=0, \quad \forall u \in \mathbb{R}^{+} .
\end{aligned}
$$

Proof If (4.7) is applied to (3.7) 2 , using (4.10), it reduces to

$$
\int_{0}^{\infty} \int_{0}^{\infty} E_{1}^{t}(s) \mathcal{G}(s, u) E_{1}^{t}(u) d s d u=\int_{0}^{\infty} \int_{0}^{\infty} E_{2}^{t}(s) \mathcal{G}(s, u) E_{2}^{t}(u) d s d u,
$$

or

$$
\int_{0}^{\infty} \int_{0}^{\infty} \mathcal{G}(s, u)\left[E_{1}^{t}(s) E_{1}^{t}(u)-E_{2}^{t}(s) E_{2}^{t}(u)\right] d s d u=0 .
$$

Now

$$
\begin{aligned}
E_{1}^{t}(s) E_{1}^{t}(u)-E_{2}^{t}(s) E_{2}^{t}(u)= & \left(E_{1}^{t}(s)-E_{2}^{t}(s)\right)\left(E_{1}^{t}(u)+E_{2}^{t}(u)\right) \\
& +E_{2}^{t}(s) E_{1}^{t}(u)-E_{2}^{t}(u) E_{1}^{t}(s) .
\end{aligned}
$$

Noting that

$$
\int_{0}^{\infty} \int_{0}^{\infty} \mathcal{G}(s, u)\left[E_{2}^{t}(s) E_{1}^{t}(u)-E_{2}^{t}(u) E_{1}^{t}(s)\right] d s d u=0,
$$


by virtue of $(3.9)_{2}$ and an interchange of integration variables, we see that (4.13) can be replaced by

$$
\int_{0}^{\infty} \int_{0}^{\infty} \mathcal{G}(s, u)\left(E_{1}^{t}(s)-E_{2}^{t}(s)\right)\left(E_{1}^{t}(u)+E_{2}^{t}(u)\right) d s d u=0 .
$$

This relationship can be written as

$$
\int_{0}^{\infty} \int_{0}^{\infty} \mathcal{G}(s, u) E_{d}^{t}(s)\left(2 E_{1}^{t}(u)+E_{d}^{t}(u)\right) d s d u=0
$$

where the history $E_{1}^{t}$ can be arbitrarily chosen (Remark 3). Therefore, (4.7) implies (4.11) while (4.11) $)_{2}$ follows from (3.9). Also, since the steps of the proof are reversible, $(4.11)_{1,2}$ imply (4.7). If we integrate $(4.11)_{2}$ over $\left[u_{1}, \infty\right)$, the result is

$$
\int_{0}^{\infty} G_{2}\left(u_{1}, s\right) E_{d}^{t}(s) d s=0
$$

for all $u_{1} \geq 0$. In particular, for $u_{1}=0$, we have

$$
\int_{0}^{\infty} G_{2}(0, s) E_{d}^{t}(s) d s=0
$$

Relation $(4.11)_{3,4}$ can be shown to be equivalent to $(4.11)_{1,2}$, using integration by parts in (4.18).

These results are derived under the constraints (4.9). For the stronger assumption (4.6) for $\tau \geq 0$, it remains of course true that (4.7) and (4.11) are equivalent. The condition (4.11) states that the linear functionals

$$
\begin{array}{ll}
\int_{0}^{\infty} \mathcal{G}(s, u) E^{t}(s) d s & =\int_{0}^{\infty} \mathcal{G}(u, s) E^{t}(s) d s, \\
\int_{0}^{\infty} \widetilde{G}(s, u) \dot{E}^{t}(s) d s & =\int_{0}^{\infty} \widetilde{G}(u, s) \dot{E}^{t}(s) d s, \quad \forall u \in \mathbb{R}^{+}
\end{array}
$$

are FMSs. One can replace $E^{t}(s)$ by $E_{r}^{t}(s)$ in $(4.20)_{1}$.

Proposition 1 can be expressed as a statement that the free energy corresponding to $G(s, u)$ is a FMS if and only if, given that (4.19) holds, then (4.18) is true for all $u_{1}>0$.

If $E_{d}^{t}$ is equivalent to the zero history, then (4.7) yields that ([2], p. 152)

$$
\tilde{\psi}\left(E_{d}^{t}, 0\right)=\int_{0}^{\infty} \int_{0}^{\infty} E_{d}^{t}(s) \mathcal{G}(s, u) E_{d}^{t}(u) d s d u=0 .
$$

Condition (4.11) yields, more generally, that if either (but not both) of the histories $E_{d}^{t}$ is replaced by an arbitrary history, $(4.21)_{2}$ still holds. 


\subsection{Some Examples}

1. The Graffi-Volterra free energy: This functional is given by

$$
\begin{aligned}
\psi_{G V}(t) & =\phi(t)-\frac{1}{2} \int_{0}^{\infty} G^{\prime}(s)\left[E_{r}^{t}(s)\right]^{2} d s \\
& =S(t)-\frac{1}{2} \int_{0}^{\infty} G^{\prime}(s)\left[E^{t}(s)\right]^{2} d s .
\end{aligned}
$$

The rate of dissipation associated with $\psi_{G V}$ has the form

$$
D(t)=\frac{1}{2} \int_{0}^{\infty} G^{\prime \prime}(s)\left[E_{r}^{t}(s)\right]^{2} d s \geq 0 .
$$

The functional $\psi_{G V}(t)$ is a free energy only if the conditions

$$
G^{\prime}(s) \leq 0, \quad G^{\prime \prime}(s) \geq 0, \quad \forall s \in \mathbb{R}^{+}
$$

hold. Relations (4.24) are true in particular for the forms of the relaxation function described in Sect. 6.

We can formally express $\psi_{G V}(t)$ in the form (3.7) by putting

$$
\mathcal{G}(s, u)=-\frac{1}{2}\left[G^{\prime}(s)+G^{\prime}(u)\right] \delta(s-u), \quad s, u \in \mathbb{R}^{+},
$$

where $\delta(s-u)$ is the singular delta function. Thus,

$$
\int_{0}^{\infty} \mathcal{G}(s, u) E_{d}^{t}(u) d u=-G^{\prime}(s) E_{d}^{t}(s) \neq 0,
$$

so that (4.11) is not obeyed and $\psi_{G V}$ is not a FMS. This is shown by another method in [2], p. 218 and earlier in [7].

2. The Dill free energy: Opting for the notation $(3.7)_{3}$ rather than $(3.7)_{1}$ which is used in [2], we put

$$
\psi_{\text {Dill }}(t)=\phi(t)+\frac{1}{2} \int_{0}^{\infty} \int_{0}^{\infty} \widetilde{G}\left(s_{1}+s_{2}\right) \dot{E}^{t}\left(s_{2}\right) \dot{E}^{t}\left(s_{1}\right) d s_{1} d s_{2}
$$

which is a free energy with rate of dissipation (cf. (3.14) 1 ) given by

$$
D_{\text {Dill }}(t)=-\int_{0}^{\infty} \int_{0}^{\infty} G^{\prime}\left(s_{1}+s_{2}\right) \dot{E}^{t}\left(s_{2}\right) \dot{E}^{t}\left(s_{1}\right) d s_{1} d s_{2},
$$

if and only if $G(\cdot)$ is completely monotonic, as defined in [7].

Thus, $\widetilde{G}(s, u)=\widetilde{G}(s+u)$ and it follows immediately from Proposition 1 that the Dill free energy is a FMS, because (4.11) has the same form as (4.6) 2,3 .

3. The functional $\psi_{F}$ : This quantity, introduced in [11], is given by

$$
\begin{gathered}
\psi_{F}(t)=\phi(t)-\frac{1}{2} \int_{0}^{\infty} \frac{\left[I_{(1)}^{t}\left(\tau, E_{r}^{t}\right)\right]^{2}}{\left.G^{\prime}(\tau)\right)} d \tau, \\
I_{(1)}^{t}\left(\tau, E_{r}^{t}\right)=\int_{0}^{\infty} G^{\prime \prime}(\tau+t) E_{r}^{t}(s) d s .
\end{gathered}
$$


Note that

$$
I_{(1)}^{t}\left(\tau, E_{d}^{t}\right)=0,
$$

so that $I_{(1)}^{t}$ as well as $I^{t}$ is a FMS. We have

$$
\mathcal{G}(s, u)=-\int_{0}^{\infty} \frac{G^{\prime \prime}(s+z) G^{\prime \prime}(u+z)}{G^{\prime}(z)} d z,
$$

and (4.11) follows from (4.30). However, it is clear by inspection of (4.29), without the use of Proposition 1, that $\psi_{F}(t)$ is a FMS.

On the other hand, the formulae (4.11) give, more readily than traditional arguments, that $\psi_{G V}$ and $\psi_{\text {Dill }}$ are FMSs.

4. The work function: This functional has the form (3.16). The version of (4.11) given by (4.18) becomes, in this case,

$$
\int_{0}^{\infty} \frac{\partial}{\partial u} G(|s-u|) E_{d}^{t}(s) d s=0, \quad \forall u \in \mathbb{R}^{+} .
$$

Observe that for $u \in \mathbb{R}^{--}$, this gives the same condition as (4.6)2,3, so that if (4.32) holds, then it is true for $u \in \mathbb{R}$. Taking the Fourier transform of this relation, using (5.13) below, together with the Convolution theorem, we see that its unique solution is $E_{d}^{t}(u)=0$, $u \in \mathbb{R}^{+}$, corresponding to singleton minimal states. Therefore, (4.32) cannot be true for relaxation functions with non-singleton minimal states, and the work function is not a FMS, as demonstrated in [7], using a different approach.

\section{Frequency Domain Quantities}

Let $\Omega$ be the complex $\omega$ plane and

$$
\begin{aligned}
& \Omega^{+}=\left\{\omega \in \Omega \mid \operatorname{Im}(\omega) \in \mathbb{R}^{+}\right\}, \\
& \Omega^{(+)}=\left\{\omega \in \Omega \mid \operatorname{Im}(\omega) \in \mathbb{R}^{++}\right\} .
\end{aligned}
$$

These define the upper half-plane including and excluding the real axis, respectively. Similarly, $\Omega^{-}, \Omega^{(-)}$are the lower half-planes including and excluding the real axis, respectively.

\subsection{Relaxation Function}

Let us define the quantities

$$
\begin{aligned}
& \widetilde{G}_{+}(\omega)=\int_{0}^{\infty} \widetilde{G}(s) e^{-i \omega s} d s=\widetilde{G}_{c}(\omega)-i \widetilde{G}_{s}(\omega), \\
& G_{+}^{\prime}(\omega)=\int_{0}^{\infty} G^{\prime}(s) e^{-i \omega s} d s=G_{c}^{\prime}(\omega)-i G_{s}^{\prime}(\omega) .
\end{aligned}
$$

It follows from (3.3) that $G_{+}^{\prime}$ and $\widetilde{G}_{+} \in L^{2}(\mathbb{R})$. Applying a partial integration in (5.2) 3 yields that

$$
G_{+}^{\prime}(\omega)=-\widetilde{G}_{0}+i \omega \widetilde{G}_{+}(\omega)
$$


so that

$$
G_{c}^{\prime}(\omega)=-\widetilde{G}_{0}+\omega \widetilde{G}_{s}(\omega), \quad G_{s}^{\prime}(\omega)=-\omega \widetilde{G}_{c}(\omega) .
$$

Note the properties of $\widetilde{G}_{+}$and $G_{+}^{\prime}$ that ([2], p. 552, for example)

$$
\begin{aligned}
\lim _{\omega \rightarrow \infty} i \omega \widetilde{G}_{+}(\omega) & =\lim _{\omega \rightarrow \infty} \omega \widetilde{G}_{s}(\omega)=\widetilde{G}_{0}, \\
\lim _{\omega \rightarrow \infty} i \omega G_{+}^{\prime}(\omega) & =\lim _{\omega \rightarrow \infty} \omega G_{s}^{\prime}(\omega)=-\lim _{\omega \rightarrow \infty} \omega^{2} \widetilde{G}_{c}(\omega)=G^{\prime}(0) .
\end{aligned}
$$

Further properties of $\widetilde{G}_{c}(\omega)$ and $G_{s}^{\prime}(\omega)$ include $[2,14]$

$$
\widetilde{G}_{c}(\omega) \geq 0, G_{s}^{\prime}(\omega) \leq 0, \quad \forall \omega \in \mathbb{R}^{++},
$$

which are consequences of the second law. We have

$$
\widetilde{G}_{0}>0, \quad G_{\infty}>0
$$

the latter relation being true for a viscoelastic solid.

Let us now discuss other properties of $G^{\prime}$, which apply also to $\widetilde{G}$. The function $G_{+}^{\prime}(\omega)$ is analytic on $\Omega^{(-)}$and, by assumption, on an open set including $\mathbb{R}$. This implies that any singularities are at least slightly off the real axis into $\Omega^{(+)}$, which in turn means that $G^{\prime}$ decays exponentially at large positive times, though perhaps weakly.

Because $G^{\prime}$ is real, we have from (5.2), for $\omega \in \Omega$,

$$
\overline{G_{+}^{\prime}(\omega)}=G_{+}^{\prime}(-\bar{\omega}),
$$

where the bar denotes complex conjugate. This constraint means that the singularities are symmetric under reflection in the positive imaginary axis. In particular, for real $\omega, \overline{G_{+}^{\prime}(\omega)}=$ $\overline{G_{+}^{\prime}}(\omega)=G_{+}^{\prime}(-\omega)$, where the bar over $G_{+}^{\prime}$ acts on the function but not the variable $\omega$.

The quantity $\overline{G_{+}^{\prime}}(\omega)$ is analytic in $\Omega^{+}$, its singularity structure being a mirror image, in the real axis, of that of $G_{+}^{\prime}(\omega)$. Thus, the singularities of $G_{s}^{\prime}(\omega)$ are in both $\Omega^{(+)}$and $\Omega^{(-)}$and are mirror images of one another. Similarly, its zeros will be mirror images of one another.

A quantity which will be of significant interest, particularly in the context of the minimum and related free energies, is

$$
H(\omega)=-\omega G_{s}^{\prime}(\omega)=\omega^{2} \widetilde{G}_{c}(\omega) \geq 0, \quad \omega \in \mathbb{R},
$$

where the inequality is a consequence of (5.6). Using (5.5) 4,5 and (5.9), one can show that

$$
H_{\infty}=\lim _{\omega \rightarrow \infty} H(\omega)=-G^{\prime}(0) \geq 0 .
$$

We assume for present purposes that $G^{\prime}(0)$ is non-zero so that $H_{\infty}$ is a finite, positive number. Then $H(\omega) \in \mathbb{R}^{++} \forall \omega \in \mathbb{R}, \omega \neq 0$, and can always be expressed as the product of two factors

$$
H(\omega)=H_{+}(\omega) H_{-}(\omega)
$$


where $H_{+}(\omega)$ has no zeros in $\Omega^{(-)}$and is analytic in $\Omega^{-}$. Similarly, $H_{-}(\omega)$ is analytic in $\Omega^{+}$with no zeros in $\Omega^{(+)}[2,10]$. We put $[2,17]$

$$
\begin{aligned}
& H_{ \pm}(\omega)=H_{\mp}(-\omega)=\overline{H_{\mp}}(\omega), \\
& H(\omega)=\left|H_{ \pm}(\omega)\right|^{2}, \quad \omega \in \mathbb{R} .
\end{aligned}
$$

A general method is outlined in $[2,17]$ for determining the factors of $H$, though for the case of discrete spectrum materials, one can deduce $H_{ \pm}$by inspection.

The factorization (5.11) is the one relevant to the minimum free energy. We shall require a much broader class of factorizations, where the property that the zeros of $H_{ \pm}(\omega)$ are in $\Omega^{ \pm}$respectively need not be true. These generate a range of free energies related to the minimum free energy, as discussed briefly in Sect. 9.

Let us define $G(s)$ on $\mathbb{R}$ by taking the even extension $G(s)=G(|s|)$, for $s \in \mathbb{R}$, so that $G^{\prime}(s)$ is an odd function. Then ([2], p. 546),

$$
G_{F}^{\prime}(\omega)=\int_{-\infty}^{\infty} G^{\prime}(|s|) e^{-i \omega s} d s=-2 i G_{s}^{\prime}(\omega)=\frac{2 i}{\omega} H(\omega)
$$

\subsection{Strain History}

We assume that the history of strain $E^{t}$ is in $L^{1}\left(R^{+}\right) \cap L^{2}\left(R^{+}\right)$. If the quantity $E^{t}(s)$ is taken to be zero for $s<0$, its Fourier transform is given by

$$
E_{+}^{t}(\omega)=\int_{0}^{\infty} E^{t}(s) e^{-i \omega s} d s .
$$

We have $E_{+}^{t}(\omega) \in L^{2}(\mathbb{R})$. This quantity is analytic on $\Omega^{(-)}$and, by assumption, on an open set including $\mathbb{R}$. For a constant history, $E^{t}(s)=E(t), s \in \mathbb{R}^{+}$, we have ([2], p. 552)

$$
E_{+}^{t}(\omega)=\frac{E(t)}{i \omega^{-}} .
$$

The Fourier transform of $E_{r}^{t}$, defined by (2.2) and zero for $s \in \mathbb{R}^{--}$therefore has the form

$$
E_{r+}^{t}(\omega)=E_{+}^{t}(\omega)-\frac{E(t)}{i \omega^{-}}
$$

The notation $\omega^{ \pm}$was introduced in [17] and used in various subsequent papers. It implies that $\omega$ is slightly off the real axis at either $\omega_{r} \pm i \epsilon$ (where $\omega_{r}$ is on the real axis). The limit $\omega \rightarrow \omega_{r}$ can be taken after integrations have been carried out. It is useful for example in applying the Plemelj formulae. We have ([2], p. 145)

$$
\frac{d}{d t} E_{+}^{t}(\omega)=\dot{E}_{+}^{t}(\omega)=-i \omega E_{+}^{t}(\omega)+E(t)=-i \omega E_{r+}^{t}(\omega) .
$$

For large $\omega$ (cf. (5.5)),

$$
E_{+}^{t}(\omega) \sim \frac{E(t)}{i \omega}, \quad E_{r+}^{t}(\omega) \sim \frac{A(t)}{\omega^{2}},
$$

where $A(t)$ is independent of $\omega$. Also, from (5.17),

$$
\dot{E}_{+}^{t}(\omega) \sim \frac{A(t)}{i \omega} .
$$




\section{Discrete Spectrum Materials}

The forms of the relaxation function and related quantities for discrete spectrum materials are specified in this section. We have

$$
\widetilde{G}(s)=\sum_{i=1}^{n} G_{i} e^{-\alpha_{i} s}
$$

where $n$ is a positive integer. The inverse decay times $\alpha_{i} \in \mathbb{R}^{+}, i=1,2, \ldots, n$ and the coefficients $G_{i}$ are also generally assumed to be positive, this being the simplest way to ensure the condition (5.6), which is clear from (6.2) 2 below. We arrange that $\alpha_{1}<\alpha_{2}<$ $\alpha_{3} \ldots$... From (5.2) $)_{1}$, we have

$$
\widetilde{G}_{+}(\omega)=\sum_{i=1}^{n} \frac{G_{i}}{\alpha_{i}+i \omega}, \quad \widetilde{G}_{c}(\omega)=\sum_{i=1}^{n} \frac{\alpha_{i} G_{i}}{\alpha_{i}^{2}+\omega^{2}}, \quad \widetilde{G}_{s}(\omega)=\omega \sum_{i=1}^{n} \frac{G_{i}}{\alpha_{i}^{2}+\omega^{2}}
$$

Relation (5.9) gives

$$
H(\omega)=\omega^{2} \sum_{i=1}^{n} \frac{\alpha_{i} G_{i}}{\alpha_{i}^{2}+\omega^{2}} \geq 0, \quad \omega \in \mathbb{R} .
$$

Let us now characterize minimal states in the context of discrete spectrum materials. Consider two states $\left(E_{1}^{t}, E_{1}(t)\right)$ and $\left(E_{2}^{t}, E_{2}(t)\right)$ obeying conditions (4.6), so that they are equivalent. Using the notation of (4.5) and (5.14), we see that (4.6) holds for all $\tau \geq 0$ if and only if

$$
E_{d}(t)=0, \quad \int_{0}^{\infty} e^{-\alpha_{j} s} E_{d}^{t}(s) d s=E_{d+}^{t}\left(-i \alpha_{j}\right)=0, \quad j=1,2, \ldots, n .
$$

Remark 4 Thus, the property that two given histories are equivalent, or in the same minimal state, is determined solely by the values of those histories in the frequency domain at $\omega=$ $-i \alpha_{j}, j=1,2, \ldots, n$.

Remark 5 General materials with only isolated singularities are discussed in Chaps. 15 and 16 of [2]. These, for many purposes, can be simplified to the case where all singularities are simple poles in $\Omega^{(+)}$.

Discrete spectrum materials are a further special case of materials with simple poles, where the poles of $\widetilde{G}_{+}(\omega)$ lie along the positive imaginary axis. It is relatively simple to generalize the results given in this work to materials with simple poles off the imaginary axis. It is also possible to merge these poles to form more general isolated singularities.

We will use discrete spectrum materials as a proxy for the more general case with only isolated singularities, to provide simpler arguments.

\section{Time Domain Theory Representations of Free Energy Functionals}

A method is developed in [20] for determining free energy functionals with desired nonnegativity properties, for the time and frequency domains. This approach allows us to choose 
a kernel for the rate of dissipation and determine from this the kernel for the free energy. In the time domain version, we have the relations [20]

$$
\widetilde{G}(s, u)=-\int_{0}^{\infty} K(z+s, z+u) d z, \quad \mathcal{G}(s, u)=-\int_{0}^{\infty} K_{12}(z+s, z+u) d z .
$$

It follows that

$$
\widetilde{G}(s)=\widetilde{G}(s, 0)=-\int_{0}^{\infty} K(z+s, z) d z=-\int_{0}^{\infty} K(z, z+s) d z .
$$

Relation $(4.11)_{3}$, for $\widetilde{G}(s, u)$ given by $(7.1)_{1}$, becomes

$$
\int_{0}^{\infty} \int_{0}^{\infty} K(z+s, z+u) \dot{E}_{d}^{t}(s) d s d z=0, \quad u \geq 0 .
$$

\section{Frequency Domain Representations of Free Energies and Dissipation Rates}

A formula equivalent to (7.3) in the frequency domain is introduced in this section, using certain results from [20] which are now summarized.

Frequency domain representations for the work function (see (3.16)) are given by ([2], p. 154)

$$
\begin{aligned}
W(t) & =\phi(t)+\frac{1}{2 \pi} \int_{-\infty}^{\infty} \frac{H(\omega)}{\omega^{2}}\left|\dot{E}_{+}^{t}(\omega)\right|^{2} d \omega \\
& =\phi(t)+\frac{1}{2 \pi} \int_{-\infty}^{\infty} H(\omega)\left|E_{r+}^{t}(\omega)\right|^{2} d \omega .
\end{aligned}
$$

Both forms are manifestly non-negative. One follows from the other by invoking (5.17).

Let us now consider free energies with general kernels, as given by $(3.7)_{3}$, with rates of dissipation specified by $(3.14)_{1}$. We define [20]

$$
Z_{+-}\left(\omega_{1}, \omega_{2}\right)=\int_{0}^{\infty} \int_{0}^{\infty} Z(s, u) e^{-i \omega_{1} s+i \omega_{2} u} d s d u
$$

where $Z(s, u)$ represents the kernel $\widetilde{G}(s, u)$ or $K(s, u)$. Note that

$$
\overline{Z_{+-}}\left(\omega_{1}, \omega_{2}\right)=Z_{+-}\left(-\omega_{1},-\omega_{2}\right)=Z_{+-}\left(\omega_{2}, \omega_{1}\right),
$$

so that $Z_{+-}(\omega, \omega)$ is real. The quantity $Z_{+-}\left(\omega_{1}, \omega_{2}\right)$ is analytic in the lower half of the $\omega_{1}$ complex plane and in the upper half of the $\omega_{2}$ plane. Inverting Fourier transforms in (8.2) yields that

$$
Z(s, u)=\frac{1}{4 \pi^{2}} \int_{-\infty}^{\infty} \int_{-\infty}^{\infty} Z_{+-}\left(\omega_{1}, \omega_{2}\right) e^{i \omega_{1} s-i \omega_{2} u} d \omega_{1} d \omega .
$$

The frequency domain version of the kernels $\mathcal{G}(s, u)$ and $\mathcal{K}(s, u)$ occurs in quadratic forms expressed in terms of $E_{r+}^{t}(\omega)$ rather than $\dot{E}_{+}^{t}(\omega)$. We can easily switch to this type of functional by using (5.17). Relations (3.15) 1 and (8.2) yield that

$$
i\left(\omega_{1}-\omega_{2}\right) \widetilde{G}_{+-}\left(\omega_{1}, \omega_{2}\right)=K_{+-}\left(\omega_{1}, \omega_{2}\right)+\widetilde{G}_{+}\left(\omega_{1}\right)+\widetilde{G}_{+}\left(\omega_{2}\right),
$$


where $\widetilde{G}_{+}(\omega)$ is defined by (5.2) $)_{1}$. From (3.11), (5.2) 1 and (8.2), it follows that [20]

$$
\widetilde{G}_{+-}\left(\omega_{1}, \omega_{2}\right) \sim \begin{cases}\frac{\widetilde{G}_{+}\left(\omega_{1}\right)}{-i \omega_{2}} & \text { as } \omega_{2} \rightarrow \infty \\ \frac{\widetilde{G}_{+}\left(\omega_{2}\right)}{i \omega_{1}} & \text { as } \omega_{1} \rightarrow \infty\end{cases}
$$

We can write $(3.7)_{3}$ and $(3.14)_{1}$ in the forms

$$
\begin{aligned}
& \psi(t)=\phi(t)+\frac{1}{8 \pi^{2}} \int_{-\infty}^{\infty} \int_{-\infty}^{\infty} \overline{\dot{E}_{+}^{t}}\left(\omega_{1}\right) \widetilde{G}_{+-}\left(\omega_{1}, \omega_{2}\right) \dot{E}_{+}^{t}\left(\omega_{2}\right) d \omega_{1} d \omega_{2}, \\
& D(t)=-\frac{1}{8 \pi^{2}} \int_{-\infty}^{\infty} \int_{-\infty}^{\infty} \overline{\dot{E}_{+}^{t}}\left(\omega_{1}\right) K_{+-}\left(\omega_{1}, \omega_{2}\right) \dot{E}_{+}^{t}\left(\omega_{2}\right) d \omega_{1} d \omega_{2} .
\end{aligned}
$$

As for the time domain expressions, we conclude from P2 and P3 (see (2.7), (2.8)) that the quadratic functionals in $(8.7)_{1}$ must be non-negative, while that in $(8.7)_{2}$ must be nonpositive. It is shown in [20], using (8.5), that

$$
\begin{aligned}
\psi(t) & =\phi(t)-\frac{i}{8 \pi^{2}} \int_{-\infty}^{\infty} \int_{-\infty}^{\infty} \frac{\overline{\dot{E}_{+}^{t}}\left(\omega_{1}\right) K_{+-}\left(\omega_{1}, \omega_{2}\right) \dot{E}_{+}^{t}\left(\omega_{2}\right)}{\omega_{1}^{+}-\omega_{2}^{-}} d \omega_{1} d \omega_{2} \\
& =\phi(t)-\frac{i}{8 \pi^{2}} \int_{-\infty}^{\infty} \int_{-\infty}^{\infty} \frac{\overline{E_{r+}^{t}}\left(\omega_{1}\right) \omega_{1} \omega_{2} K_{+-}\left(\omega_{1}, \omega_{2}\right) E_{r+}^{t}\left(\omega_{2}\right)}{\omega_{1}^{+}-\omega_{2}^{-}} d \omega_{1} d \omega_{2},
\end{aligned}
$$

where one form follows from the other by using (5.17). Relation $(8.8)_{1}$ can also be derived from (7.1), using (8.4). The quantities $\omega^{ \pm}$are discussed briefly after (5.16). The double use of this notation in the denominator specifies the procedure, depending on which integration is carried out first ([20] and earlier work, [2], p. 250). It is shown in [20] that

$$
\begin{aligned}
K_{+-}(\omega, \omega) & =-2 \frac{H(\omega)}{\omega^{2}}=-2 \widetilde{G}_{c}(\omega)=-\widetilde{G}_{+}(\omega)-\overline{\widetilde{G}_{+}}(\omega) \\
& =-\frac{G_{+}^{\prime}(\omega)}{i \omega}+\frac{\overline{G_{+}^{\prime}}(\omega)}{i \omega}, \quad \omega \in \mathbb{R},
\end{aligned}
$$

where $(5.2)_{1},(5.3)$ and (5.9) have also been invoked.

Taking the Fourier transform of the free variable $u$ in $(4.11)_{4}$, and applying Parseval's formula to the integrated product, we obtain

$$
\int_{-\infty}^{\infty} \widetilde{G}_{+-}\left(\omega, \omega_{1}\right) \dot{E}_{d+}^{t}\left(\omega_{1}\right) d \omega_{1}=0, \quad \omega \in \mathbb{R}
$$

where $E_{d+}^{t}$ is the Fourier transform of $E_{d}^{t}$, defined by (4.5); see also (6.4). Then, (8.5) gives

$$
\begin{aligned}
& \int_{-\infty}^{\infty} \frac{K_{+-}\left(\omega, \omega_{1}\right) \dot{E}_{d+}^{t}\left(\omega_{1}\right)}{\omega_{1}-\omega^{ \pm}} d \omega_{1} \\
& \quad=-\widetilde{G}_{+}(\omega) \int_{-\infty}^{\infty} \frac{\dot{E}_{d+}^{t}\left(\omega_{1}\right)}{\omega_{1}-\omega^{ \pm}} d \omega_{1}-\int_{-\infty}^{\infty} \frac{\overline{\widetilde{G}_{+}}\left(\omega_{1}\right) \dot{E}_{d+}^{t}\left(\omega_{1}\right)}{\omega_{1}-\omega^{ \pm}} d \omega_{1}, \quad \forall \omega \in \mathbb{R} .
\end{aligned}
$$


The versions with $\omega^{+}$and $\omega^{-}$will be shown to be equivalent. Consider the first integral on the right of (8.11), for $\omega^{+}$. Closing the contour on $\Omega^{(-)}$, it is clear that

$$
\int_{-\infty}^{\infty} \frac{\dot{E}_{d+}^{t}\left(\omega_{1}\right)}{\omega_{1}-\omega^{+}} d \omega_{1}=0
$$

Condition (4.6) 3 has the form [21]

$$
I_{d}^{t}(\tau)=\frac{1}{2 \pi} \int_{-\infty}^{\infty}\left[\widetilde{G}_{+}\left(\omega_{1}\right)+\lambda \widetilde{G}_{+}\left(\omega_{1}\right)\right] \dot{E}_{d+}^{t}\left(\omega_{1}\right) e^{-i \omega_{1} \tau} d \omega_{1}=0, \quad \tau \geq 0
$$

where $\lambda$ is any complex constant. It follows that

$$
\int_{0}^{\infty} I_{d}^{t}(\tau) e^{-i \omega \tau} d \tau=I_{d+}^{t}(\omega)=0, \quad \omega \in \mathbb{R}
$$

From the complex conjugate of this relationship, $\overline{I_{d+}^{t}}(\omega)=0$, we deduce that

$$
\int_{-\infty}^{\infty} \frac{\left[\widetilde{\widetilde{G}_{+}}\left(\omega_{1}\right)+\lambda \widetilde{G}_{+}\left(\omega_{1}\right)\right] \dot{E}_{d+}^{t}\left(\omega_{1}\right)}{\omega_{1}-\omega^{+}} d \omega_{1}=0, \quad \forall \omega \in \mathbb{R}
$$

where the choice $\omega^{+}$is dictated by convergence requirements. The enforcement of (8.15) ensures that $\dot{E}_{d+}^{t}(\omega)$ is the Fourier transform of a history equivalent to zero. Combining (8.12) with (8.15) for $\lambda=0$, we obtain

$$
\int_{-\infty}^{\infty} \frac{K_{+-}\left(\omega, \omega_{1}\right) \dot{E}_{d+}^{t}\left(\omega_{1}\right)}{\omega_{1}-\omega^{+}} d \omega_{1}=0
$$

From (8.16), (8.9) and the Plemelj formulae, we obtain

$$
\frac{1}{2 \pi i} \int_{-\infty}^{\infty} \frac{K_{+-}\left(\omega, \omega_{1}\right) \dot{E}_{d+}^{t}\left(\omega_{1}\right)}{\omega_{1}-\omega^{-}} d \omega_{1}=2 \frac{H(\omega)}{\omega^{2}} \dot{E}_{d+}^{t}(\omega),
$$

which is equal to the right-hand side of (8.11), for $\omega^{-}$in the denominator. Either (8.16) or (8.17) express condition (8.10) or (4.11).

\section{The Minimum and Related Free Energies}

A particularly interesting example of the formalism discussed in Sect. 8 is now briefly summarized.

It is shown in $[15,18]$ that, for materials with only isolated singularities, the quantity $H(\omega)$ is a rational function and has various factorizations other than (5.11), denoted by

$$
H(\omega)=H_{+}^{f}(\omega) H_{-}^{f}(\omega), \quad H_{ \pm}^{f}(\omega)=H_{\mp}^{f}(-\omega)=\overline{H_{\mp}^{f}}(\omega),
$$

where $f$ is an identification label distinguishing a particular factorization. These are obtained by exchanging the zeros of $H_{+}(\omega)$ and $H_{-}(\omega)$, leaving the singularities unchanged. Thus, $H_{-}^{f}(\omega)$ has all its singularities in $\Omega^{(-)}$. We have, by virtue of (5.17),

$$
H_{-}^{f}(\omega) E_{r+}^{t}(\omega)=i \frac{H_{-}^{f}(\omega)}{\omega} \dot{E}_{+}^{t}(\omega)=p_{-}^{f t}(\omega)-p_{+}^{f t}(\omega),
$$


where

$$
\begin{aligned}
p_{ \pm}^{f t}(\omega) & =\frac{1}{2 \pi i} \int_{-\infty}^{\infty} \frac{H_{-}^{f}\left(\omega^{\prime}\right) E_{r+}^{t}\left(\omega^{\prime}\right)}{\omega^{\prime}-\omega^{\mp}} d \omega^{\prime} \\
& =\frac{1}{2 \pi} \int_{-\infty}^{\infty} \frac{H_{-}^{f}\left(\omega^{\prime}\right) \dot{E}_{+}^{t}(\omega)}{\omega^{\prime}\left(\omega^{\prime}-\omega^{\mp}\right)} d \omega^{\prime} .
\end{aligned}
$$

Each factorization yields a (usually) different free energy of the form

$$
\psi_{f}(t)=\phi(t)+\frac{1}{2 \pi} \int_{-\infty}^{\infty}\left|p_{-}^{f t}(\omega)\right|^{2} d \omega .
$$

Remark 6 All the quantities $p_{-}^{f t}(\omega)$ can be shown to be FMSs ([2], p. 340). In particular, the basic quantity $p_{-}^{t}(\omega)$, corresponding to $f=1$, which is defined for all materials, including those with branch points, is an FMS ([2], p. 253 and [10]). Relation (9.4) for $p_{-}^{t}(\omega)$ yields the minimum free energy. It follows from the fact that the $p_{-}^{f t}(\omega)$ are FMSs that all the free energies $\psi_{f}(t)$ also have this property.

The quantity $W(t)$, given by (8.1), can be expressed as ([2], p. 353)

$$
W(t)=\phi(t)+\frac{1}{2 \pi} \int_{-\infty}^{\infty}\left[\left|p_{-}^{f t}(\omega)\right|^{2}+\left|p_{+}^{f t}(\omega)\right|^{2}\right] d \omega .
$$

It can be shown that the rate of dissipation associated with $\psi_{f}(t)$ is

$$
D_{f}(t)=\left|K_{f}(t)\right|^{2},
$$

where

$$
K_{f}(t)=-\frac{1}{2 \pi i} \int_{-\infty}^{\infty} \frac{H_{-}^{f}(\omega)}{\omega} \dot{E}_{+}^{t}(\omega) d \omega=\frac{1}{2 \pi} \int_{-\infty}^{\infty} H_{-}^{f}(\omega) E_{r+}^{t}(\omega) d \omega .
$$

We can write $\psi_{f}(t)$ in the form (see $\left.(8.8)\right)[2,18]$

$$
\begin{aligned}
\psi_{f}(t) & =\phi(t)+\frac{i}{4 \pi^{2}} \int_{-\infty}^{\infty} \int_{-\infty}^{\infty} \frac{\overline{\dot{E}_{+}^{t}}\left(\omega_{1}\right) H_{+}^{f}\left(\omega_{1}\right) H_{-}^{f}\left(\omega_{2}\right) \dot{E}_{+}^{t}\left(\omega_{2}\right)}{\omega_{1} \omega_{2}\left(\omega_{1}^{+}-\omega_{2}^{-}\right)} d \omega_{1} d \omega_{2} \\
& =\phi(t)+\frac{i}{4 \pi^{2}} \int_{-\infty}^{\infty} \int_{-\infty}^{\infty} \frac{\overline{E_{r+}^{t}}\left(\omega_{1}\right) H_{+}^{f}\left(\omega_{1}\right) H_{-}^{f}\left(\omega_{2}\right) E_{r+}^{t}\left(\omega_{2}\right)}{\omega_{1}^{+}-\omega_{2}^{-}} d \omega_{1} d \omega_{2}
\end{aligned}
$$

while $D_{f}(t)$, given by (9.6), can be expressed as (see $\left.(8.7)_{2}\right)$

$$
\begin{aligned}
D_{f}(t) & =\frac{1}{4 \pi^{2}} \int_{-\infty}^{\infty} \overline{\dot{E}_{+}^{t}}\left(\omega_{1}\right) \frac{H_{+}^{f}\left(\omega_{1}\right) H_{-}^{f}\left(\omega_{2}\right)}{\omega_{1} \omega_{2}} \dot{E}_{+}^{t}\left(\omega_{2}\right) d \omega_{1} d \omega_{2} \\
& =\frac{1}{4 \pi^{2}} \int_{-\infty}^{\infty} \overline{E_{r+}^{t}}\left(\omega_{1}\right) H_{+}^{f}\left(\omega_{1}\right) H_{-}^{f}\left(\omega_{2}\right) E_{r+}^{t}\left(\omega_{2}\right) d \omega_{1} d \omega_{2} .
\end{aligned}
$$

Comparing (9.9) and (8.7) 2 , we see that

$$
K_{+-}\left(\omega_{1}, \omega_{2}\right)=-2 \frac{H_{+}^{f}\left(\omega_{1}\right) H_{-}^{f}\left(\omega_{2}\right)}{\omega_{1} \omega_{2}},
$$


and (8.16) is true if and only if

$$
p_{d-}^{f t}(\omega)=\frac{1}{2 \pi} \int_{-\infty}^{\infty} \frac{H_{-}^{f}\left(\omega^{\prime}\right) \dot{E}_{d+}^{t}(\omega \prime)}{\omega^{\prime}\left(\omega^{\prime}-\omega^{+}\right)} d \omega^{\prime}=0
$$

which is the property noted in Remark 6.

All the free energies $\psi_{f}(t)$ are on the boundary of the convex set of free energies associated with a given state of the material, in the sense that the rate of dissipation given by (9.9) is a non-negative rather than a positive definite functional. The factorization (5.11) corresponds to $f=1$ and yields the minimum free energy $\psi_{m}(t)$. Each exchange of zeros, starting from these factors, can be shown to yield a free energy which is greater than or equal to the previous quantity ([15], [2], p. 363).

Remark 7 A particularly interesting one is obtained by interchanging all the zeros. This can be identified as the maximum free energy among all those that are FMSs. It is less than the work function, which is not a FMS for materials with only isolated singularities, as shown in Sect. 4.1. Furthermore, it is not necessarily greater than the Graffi-Volterra free energy, since the latter is also not a FMS [3].

There are several (indeed many, for a large number of isolated singularities) different zero exchange pathways leading from the minimum to the maximum free energy.

The most general free energy and rate of dissipation arising from these factorizations is given by

$$
\psi(t)=\sum_{f=1}^{N} \lambda_{f} \psi_{f}(t), \quad D(t)=\sum_{f=1}^{N} \lambda_{f} D_{f}(t), \quad \sum_{f=1}^{N} \lambda_{f}=1, \lambda_{f} \geq 0 .
$$

A particular case of this linear form is the free energy proposed in [18].

We now consider a generalization of the example discussed in this section.

\section{Product Formulae in the Time and Frequency Domains}

\subsection{The Time Domain}

Consider the form [20]

$$
K(s, u)=-\sum_{i, j=1}^{m} \int_{0}^{\infty} A_{i j} k_{i}(s) k_{j}(u) d u
$$

where $k_{i} \in L^{1}\left(\mathbb{R}^{+}\right) \cap L^{2}\left(\mathbb{R}^{+}\right), i=1,2, \ldots, m$ and where $A_{i j}, i, j=1,2, \ldots, m$ are the components of a non-negative matrix.

Remark 8 It is assumed that the $k_{i}(u)$ vary independently of each other as $\mathrm{u}$ changes. In particular, they are not proportional to one another. Indeed, if some were proportional, they could be merged in the summation of (10.1). 
The rate of dissipation (3.14) becomes

$$
D(t)=\frac{1}{2} \sum_{i, j=1}^{m} \int_{0}^{\infty} \int_{0}^{\infty}\left[\dot{E}^{t}(s) A_{i j} k_{i}(s) k_{j}(u) \dot{E}^{t}(u)\right] d s d u \geq 0 .
$$

It follows from $(7.1)_{1}$ that

$$
\begin{aligned}
\psi(t)= & \phi(t) \\
& +\frac{1}{2} \int_{0}^{\infty} \int_{0}^{\infty} \int_{0}^{\infty} \dot{E}^{t}(s) \sum_{i, j=1}^{m} A_{i j} k_{i}(z+s) k_{j}(z+u) d z \dot{E}^{t}(u) d s d u .
\end{aligned}
$$

Also, from (7.2),

$$
\widetilde{G}(s)=\int_{0}^{\infty} \sum_{i, j=1}^{m} A_{i j} k_{i}(z) k_{j}(z+s) d z .
$$

The condition that $\dot{E}_{d}^{t}$ is equivalent to the zero history takes the form

$$
\sum_{i, j=1}^{m} \int_{0}^{\infty} A_{i j} k_{i}(z)\left[\int_{0}^{\infty} k_{j}(z+s+u) \dot{E}_{d}^{t}(s) d s\right] d z=0, \quad \forall u \geq 0
$$

by virtue of (10.4) and (4.6). Recalling Remark 8, we see that since (10.5) holds for arbitrary $u$, the individual constraints

$$
\int_{0}^{\infty} k_{i}(y+s) \dot{E}_{d}^{t}(s) d s=0, \quad \forall y \geq 0, i=1,2, \ldots, m
$$

must be obeyed. Condition (7.3) becomes, for $K(s, u)$ given by (10.1),

$$
\begin{aligned}
& \sum_{i, j=1}^{m} \int_{0}^{\infty} \int_{0}^{\infty} A_{i j} k_{i}(z+s) k_{j}(z+u) \dot{E}_{d}^{t}(s) d s d z \\
& \quad=\sum_{i, j=1}^{m} \int_{0}^{\infty} A_{i j} k_{j}(z+u)\left[\int_{0}^{\infty} k_{i}(z+s) \dot{E}_{d}^{t}(s) d s\right] d z=0, \quad u \geq 0,
\end{aligned}
$$

which is true by virtue of (10.6). Conversely, since $u$ is arbitrary, we see that (10.7) implies the conditions (10.6).

Relation (10.7) is equivalent to the statement that any free energy functional with rate of dissipation kernel given by (10.1) is a FMS, by virtue of Proposition 1. The conditions (10.6) are a statement that the quantities

$$
\int_{0}^{\infty} k_{i}(z+s) \dot{E}^{t}(s) d s, \quad \forall z \geq 0, i=1,2, \ldots, m,
$$

are FMSs.

1050 


\subsection{The Frequency Domain}

We consider the forms

$$
K_{+-}\left(\omega_{1}, \omega_{2}\right)=-\sum_{i, j=1}^{m} A_{i j} k_{i+}\left(\omega_{1}\right) k_{j-}\left(\omega_{2}\right)=-\sum_{i, j=1}^{m} A_{i j} \overline{k_{i-}}\left(\omega_{1}\right) k_{j-}\left(\omega_{2}\right),
$$

where

$$
k_{i+}(\omega)=\int_{0}^{\infty} k_{i}(s) e^{-i \omega s} d s, \quad k_{i-}(\omega)=\overline{k_{i+}}(\omega),
$$

and $A_{i j}$ are the components of the matrix introduced in (10.1). The quantities $k_{i-}(\omega)$ have all their singularities in $\Omega^{(-)}$and are analytic on an open set including $\Omega^{(+)}$, just as for the functions $H_{-}^{f}(\omega)$, introduced in (9.1). Recalling (9.2), we put

$$
\begin{aligned}
& i \frac{k_{i-}(\omega)}{\omega} \dot{E}_{+}^{t}(\omega)=q_{i-}^{t}(\omega)-q_{i+}^{t}(\omega), \\
& q_{i \pm}^{t}(\omega)=\frac{1}{2 \pi i} \int_{-\infty}^{\infty} \frac{k_{i-}\left(\omega^{\prime}\right) \dot{E}_{+}^{t}\left(\omega^{\prime}\right)}{\omega^{\prime}-\omega^{\mp}} d \omega^{\prime}, i=1,2 \ldots, m .
\end{aligned}
$$

Condition (8.9) 1 gives that

$$
\sum_{i, j=1}^{m} A_{i j} \overline{k_{i-}}(\omega) k_{j-}(\omega)=2 \frac{H(\omega)}{\omega^{2}}
$$

This equation implies that the singularities of $k_{j \pm}(\omega)$ are a subset (or the full set) of those in $H(\omega)$. The possibility of extra singularities occurring in individual terms, which drop out of the summation due to cancellation between terms, is excluded by assumption.

Relations $(8.7)_{2}$ and (8.8) yield

$$
\begin{aligned}
& D(t)=\frac{1}{8 \pi^{2}} \int_{-\infty}^{\infty} \int_{-\infty}^{\infty} \overline{\dot{E}_{+}^{t}}\left(\omega_{1}\right) \sum_{i, j=1}^{m} A_{i j} \overline{k_{i-}}\left(\omega_{1}\right) k_{j-}\left(\omega_{2}\right) \dot{E}_{+}^{t}\left(\omega_{2}\right) d \omega_{1} d \omega_{2}, \\
& \psi(t)=\phi(t)+\frac{i}{8 \pi^{2}} \int_{-\infty}^{\infty} \int_{-\infty}^{\infty} \frac{\overline{\dot{E}_{+}^{t}}\left(\omega_{1}\right) \sum_{i, j=1}^{m} A_{i j} \overline{k_{i-}}\left(\omega_{1}\right) k_{j-}\left(\omega_{2}\right) \dot{E}_{+}^{t}\left(\omega_{2}\right)}{\omega_{1}^{+}-\omega_{2}^{-}} d \omega_{1} d \omega_{2} .
\end{aligned}
$$

Remark 9 In the case where $m=1$, we have, putting $A_{11}=1$,

$$
K_{+-}\left(\omega_{1}, \omega_{2}\right)=-k_{+}\left(\omega_{1}\right) k_{-}\left(\omega_{2}\right)
$$

and relation (10.12) becomes

$$
k_{+}(\omega) k_{-}(\omega)=\left|k_{-}(\omega)\right|^{2}=2 \frac{H(\omega)}{\omega^{2}} .
$$

Relation (10.15) is of course the factorization problem for $H(\omega)$, discussed extensively in previous work [2]. The solution is given by the factorizations (9.1) (which include (5.11) 
relating to the minimum free energy). Thus, the solution of (10.15) is non-unique. We put

$$
k_{-}(\omega)=\sqrt{2} \frac{H_{-}^{f}(\omega)}{\omega} .
$$

It follows from (10.14) that $K_{+-}\left(\omega_{1}, \omega_{2}\right)$ is given by (9.10). The rate of dissipation $D_{f}(t)$ is (9.9) and $\psi_{f}(t)$ has the form $(9.8)_{1}$.

The case $m=1$ is therefore precisely that discussed in Sect. 9. For $m>1$, which is now considered, we are dealing with more general classes of free energies.

\section{Proposition 2 The quantities}

$$
q_{j-}^{t}(\omega)=\frac{1}{2 \pi i} \int_{-\infty}^{\infty} \frac{k_{j-}\left(\omega_{1}\right) \dot{E}_{+}^{t}\left(\omega_{1}\right)}{\omega_{1}-\omega^{+}} d \omega_{1}, \quad j=1,2, \ldots, m,
$$

are FMSs.

This is a generalization of the properties noted in Remark 6.

Proof Putting $\lambda=1$ in (8.13), we determine that the quantity $I_{d}^{t}(\tau)$ has the form

$$
I_{d}^{t}(\tau)=\frac{1}{\pi} \int_{-\infty}^{\infty} \frac{H(\omega)}{\omega^{2}} \dot{E}_{d+}^{t}(\omega) e^{-i \omega \tau} d \omega
$$

Substituting (10.12) for $H(\omega)$, we find that

$$
I_{d}^{t}(\tau)=\frac{1}{2 \pi} \int_{-\infty}^{\infty} \sum_{i, j=1}^{m} A_{i j} \overline{k_{i-}}(\omega) k_{j-}(\omega) \dot{E}_{d+}^{t}(\omega) e^{-i \omega \tau} d \omega
$$

so that, from $(10.11)_{1}$,

$$
I_{d}^{t}(\tau)=-\frac{i}{2 \pi} \int_{-\infty}^{\infty} \sum_{i, j=1}^{m} A_{i j} \overline{k_{i-}}(\omega)\left[q_{d j-}^{t}(\omega)-q_{d j+}^{t}(\omega)\right] e^{-i \omega \tau} d \omega .
$$

Observe that $\overline{k_{i-}}(z), q_{j+}^{t}(z)$ and $e^{-i z \tau}(\tau>0)$ are analytic functions in the lower half-plane $z \in \Omega^{(-)}$. Their product converges strongly to zero at infinity, so that, by Cauchy's theorem, (10.20) reduces to

$$
I_{d}^{t}(\tau)=-\frac{i}{2 \pi} \int_{-\infty}^{\infty} \sum_{i, j=1}^{m} A_{i j} \overline{k_{i-}}(\omega) q_{d j-}^{t}(\omega) e^{-i \omega \tau} d \omega .
$$

We now wish to show that

$$
I_{d}^{t}(\tau)=0 \quad \forall \tau \geq 0 \quad \Longleftrightarrow \quad q_{d i-}^{t}(\omega)=0, \quad i=1,2, \ldots m, \forall \omega \in \mathbb{R} .
$$

The statement relating to the left pointing arrow of (10.22) follows trivially from (10.21). Let us consider the statement relating to the right arrow. We see from $(10.11)_{2}$, by closing the contour on $\Omega^{(-)}$, that the singularities of $q_{d i-}^{t}(\omega)$ are those of $k_{i-}(\omega)$ in $\Omega^{(-)}$. Also, $\dot{E}_{d+}^{t}(\omega)$, only occurs evaluated at the singularities of the $k_{i-}(\omega)$. The quantities $q_{i-}^{t}(\omega)$ are homogeneously linear in these quantities. By virtue of (6.4), we have that $(10.22)_{1}$ implies that all the $E_{d-}^{t}\left(-i \alpha_{i}\right)$ vanish, so that $(10.22)_{2}$ is true, which yields (10.17). 
Proposition 3 Any free energy of the form $(10.13)_{2}$ is a FMS.

Proof Relation (8.16) takes the form

$$
\sum_{i, j=1}^{m} A_{i j} \overline{k_{i-}}(\omega) \int_{-\infty}^{\infty} \frac{k_{j-}\left(\omega_{1}\right) \dot{E}_{d+}^{t}\left(\omega_{1}\right)}{\omega_{1}-\omega^{+}} d \omega_{1}=0 .
$$

This is always satisfied for $\dot{E}_{d+}^{t}$ equivalent to the zero history, by virtue of Proposition 2 . Thus, $(10.13)_{2}$ is a FMS.

These results show that for all materials described by (10.1) or (10.9), the resulting free energy is a FMS. Proposition 3 is the basis for the conjecture in Remark 1.

We note that Parseval's formula, applied to (10.6), gives (cf. (8.13))

$$
\int_{-\infty}^{\infty} k_{i-}(\omega) \dot{E}_{d+}^{t}(\omega) e^{-i \omega y} d \omega=0, \quad \forall y \geq 0, i=1,2 \ldots, m .
$$

Taking the Fourier transform of the complex conjugate of this relation gives

$$
q_{d i-}^{t}(\omega)=\frac{1}{2 \pi i} \int_{-\infty}^{\infty} \frac{k_{i-}\left(\omega_{1}\right) \dot{E}_{d+}^{t}\left(\omega_{1}\right)}{\omega_{1}-\omega^{+}} d \omega_{1}=0, \quad i=1,2, \ldots, m,
$$

where $\omega^{+}$is the choice required for convergence.

Thus, the results of Sects. 10.1 and 10.2 are in agreement.

An immediate example is obtained by taking $m=N$ in (9.12) and $A_{i j}=\delta_{i j}$, $i, j=1,2, \ldots, m$, where $\delta_{i j}$ is the Kronecker delta. We put

$$
k_{i-}(\omega)=k_{f-}(\omega)=\frac{\sqrt{2 \lambda_{f}} H_{-}^{f}(\omega)}{\omega},
$$

giving (see (9.10))

$$
K_{+-}\left(\omega_{1}, \omega_{2}\right)=-2 \sum_{f=1}^{N} \lambda_{f} \frac{H_{+}^{f}\left(\omega_{1}\right) H_{-}^{f}\left(\omega_{2}\right)}{\omega_{1} \omega_{2}} .
$$

Then, the properties specified in Remark 6, given explicitly by (9.12), yields Propositions 2 and 3 .

We now consider a deeper example, which generalizes that introduced in [20]. Let us assume that $\widetilde{G}(s)$ can be decomposed into $m$ components $\widetilde{G}_{i}(s), i=1,2, \ldots, m$, so that

$$
\widetilde{G}(s)=\sum_{i=1}^{m} \widetilde{G}_{i}(s) .
$$

Remark 10 Each $\widetilde{G}_{i}(s)$ is assumed to be the relaxation function of a well-defined material, obeying the laws of thermodynamics, in particular (5.6). The term sub-material was used in [20] for each of these. The strain function is assumed to be the same in each sub-material.

We put

$$
\widetilde{G}_{i}(s)=\int_{0}^{\infty} k_{i}(z) k_{i}(z+s) d z, \quad i=1,2, \ldots m
$$


yielding a relation of the form (10.4), with $A_{i j}=\delta_{i j}, i, j=1,2, \ldots, m$. For each $i,(10.29)$ can be regarded as a non-linear integral equation for $k_{i}(s)$ in terms of the known quantity $\widetilde{G}_{i}(s)$.

The quantity $H(\omega)$ can be decomposed into $m$ components $H_{i}(\omega), i=1,2, \ldots, m$, where

$$
H(\omega)=\sum_{i=1}^{m} H_{i}(\omega),
$$

where each $H_{i}(\omega)$ is related to $\widetilde{G}_{i}(s)$ in accordance with (5.9) and in particular is nonnegative for $\omega \in \mathbb{R}$. It is the quantity $H(\omega)$ for the sub-material with relaxation function $\widetilde{G}_{i}(s)$.

Under our assumptions, we have $\widetilde{G}(s)$ given by (6.1). Let us define $m$ vectors of $n$ dimensions, with components $\mu_{i j} \geq 0, i=1,2, \ldots, m$ and $j=1,2, \ldots, n$, putting

$$
\widetilde{G}_{i}(s)=\sum_{j=1}^{n} \mu_{i j} G_{j} e^{-\alpha_{j} s}, \quad i=1,2, \ldots, m,
$$

where

$$
\begin{aligned}
& \mu_{i j} \geq 0, \quad i=1,2, \ldots, m, j=1,2, \ldots, n, \\
& \sum_{i=1}^{m} \mu_{i j}=1, \quad j=1,2, \ldots, n .
\end{aligned}
$$

The second property follows from (10.28) and (6.1). Corresponding to (10.31), we have

$$
H_{i}(\omega)=\omega^{2} \sum_{j=1}^{m} \frac{\mu_{i j} \alpha_{j} G_{j}}{\alpha_{j}^{2}+\omega^{2}} .
$$

For example, we could take $\widetilde{G}_{i}(\omega)$ to be a partial sum of the terms in (6.1) [20]

$$
\widetilde{G}_{i}(s)=\sum_{k=n_{i}}^{m_{i}} G_{k} e^{-\alpha_{k} s}, \quad n \geq m_{i}>n_{i} \geq 1,
$$

which corresponds to

$$
\mu_{i j}= \begin{cases}0, & 1 \leq j \leq n_{i}, n \geq j \geq m_{i}>n_{i} \\ 1, & j \in\left[n_{i}, m_{i}\right],\end{cases}
$$

where the sets $\left[n_{i}, m_{i}\right]$ are disjoint, with the property that $\bigcup_{i=1}^{m}\left[n_{i}, m_{i}\right]=[1, m]$.

In general, each $H_{i}(\omega)$ can be factorized as in (9.1). We will exclude the label $f$ for simplicity and put

$$
H_{i}(\omega)=H_{i+}(\omega) H_{i-}(\omega), \quad H_{i \pm}(\omega)=H_{i \mp}(-\omega)=\overline{H_{i \mp}}(\omega) .
$$

For each $i$, we put

$$
k_{i-}(\omega)=\sqrt{2} \frac{H_{i-}(\omega)}{\omega},
$$


and (10.30) can be written as

$$
\sum_{i=1}^{m} \overline{k_{i-}}(\omega) k_{i-}(\omega)=2 \sum_{i=1}^{m} \frac{H_{i+}(\omega) H_{i-}(\omega)}{\omega^{2}}=2 \frac{H(\omega)}{\omega^{2}},
$$

where $H_{i \pm}(\omega)$ are a particular pair of factors of $H_{i}(\omega)$. Thus, we have

$$
K_{+-}\left(\omega_{1}, \omega_{2}\right)=-2 \sum_{i=1}^{m} \frac{H_{i+}\left(\omega_{1}\right) H_{i-}\left(\omega_{2}\right)}{\omega_{1} \omega_{2}} .
$$

This relation resembles (10.27) but the factors have quite different meanings. If (10.39) is inserted into $(8.7)_{2}$, we obtain

$$
\begin{aligned}
& D(t)=\sum_{i=1}^{m}\left|K_{i}(t)\right|^{2}, \\
& K_{i}(t)=-\frac{1}{2 \pi i} \int_{-\infty}^{\infty} \frac{H_{i-}(\omega)}{\omega} E_{r+}^{t}(\omega) d \omega .
\end{aligned}
$$

These relations are analogous to (9.6) and (9.7). Substituting (10.39) into (8.8) gives a sum of terms identical to (9.8) but with $H_{i \pm}$ replacing $H_{ \pm}^{f}$. These terms can be re-expressed in the form (9.4) with the same replacements. The free energy can therefore be written as

$$
\begin{aligned}
& \psi(t)=\phi(t)+\frac{1}{2 \pi} \sum_{i=1}^{m} \int_{-\infty}^{\infty}\left|p_{i-}^{t}(\omega)\right|^{2} d \omega, \\
& p_{i-}^{t}(\omega)=\frac{1}{2 \pi i} \int_{-\infty}^{\infty} \frac{H_{i-}\left(\omega^{\prime}\right) E_{r+}^{t}\left(\omega^{\prime}\right)}{\omega^{\prime}-\omega^{+}} d \omega^{\prime} .
\end{aligned}
$$

The functional $\psi(t)$ can be shown to have the properties of a free energy, as in [20]. Also, it follows from Proposition 3 that $\psi(t)$ is a FMS.

\section{Ethical Statement}

Funding: G. Amendola has been supported by the University of Pisa, M. Fabrizio by the University of Bologna, and both by the Gruppo Nazionale di Fisica Matematica of the Istituto Nazionale di Alta Matematica; J.M. Golden has been supported by the Dublin Institute of Technology.

Conflict of interest The authors declare that they have no conflict of interest.

\section{References}

1. Amendola, G., Fabrizio, M., Golden, J.M.: Free energies in a general non-local theory of a material with memory. Math. Models Methods Appl. Sci. 24, 1037-1090 (2014)

2. Amendola, G., Fabrizio, M., Golden, J.M.: Thermodynamics of Materials with Memory: Theory and Applications. Springer, New York (2012)

3. Amendola, G., Fabrizio, M., Golden, J.M.: Algebraic and numerical exploration of free energies for materials with memory. Electr. J. Diff. Eq. 72, 1-42 (2015) 
4. Berti, V., Gentili, G.: The minimum free energy for isothermal dielectrics with memory. J. Non-Equilib. Thermodyn. 24, 154-176 (1999)

5. Coleman, B.D.: Thermodynamics of materials with memory. Arch. Ration. Mech. Anal. 17, 1-45 (1964)

6. Coleman, B.D., Owen, D.: A mathematical foundation for thermodynamics. Arch. Ration. Mech. Anal. 54, 1-104 (1974)

7. Del Piero, G., Deseri, L.: On the analytic expression of the free energy in linear viscoelasticity. J. Elast. 43, 247-278 (1996)

8. Del Piero, G., Deseri, L.: On the concepts of state and free energy in linear viscoelasticity. Arch. Ration. Mech. Anal. 138, 1-35 (1997)

9. Deseri, L., Di Paola, M., Zingales, M.: Free energy and states of fractional-order hereditariness. Int. J. Solids Struct. 51(18), 3156-3167 (2014). doi:10.1016/j.ijsolstr.2014.05.008

10. Deseri, L., Gentili, G., Golden, J.M.: An explicit formula for the minimum free energy in linear viscoelasticity. J. Elast. 54, 141-185 (1999)

11. Deseri, L., Fabrizio, M., Golden, J.M.: On the concept of a minimal state in viscoelasticity: new free energies and applications to $\mathrm{PDE}_{S}$. Arch. Ration. Mech. Anal. 181, 43-96 (2006)

12. Deseri, L., Golden, J.M.: The minimum free energy for continuous spectrum materials. SIAM J. Appl. Math. 67, 869-892 (2007)

13. Deseri, L., Zingales, M., Pollaci, P.: The state of fractional hereditary materials (FHM). Differ. Equ. Dyn. Syst. B 19(7), 2065-2089 (2014). doi:10.3934/dcdsb.2014.19.2065

14. Fabrizio, M., Morro, A.: Mathematical Problems in Linear Viscoelasticity. SIAM, Philadelphia (1992)

15. Fabrizio, M., Golden, J.M.: Maximum and minimum free energies for a linear viscoelastic material. Q. Appl. Math. 60, 341-381 (2002)

16. Fabrizio, M., Gentili, G., Golden, J.M.: Free energies for linear non-isothermal materials with memory. Math. Comput. Model. 39, 219-253 (2004)

17. Golden, J.M.: Free energies in the frequency domain: the scalar case. Q. Appl. Math. 58, 127-150 (2000)

18. Golden, J.M.: A proposal concerning the physical rate of dissipation in materials with memory. Q. Appl. Math. 63, 117-155 (2005)

19. Golden, J.M.: A proposal concerning the physical dissipation of materials with memory: the nonisothermal case. Math. Mech. Solids 12, 403-449 (2007)

20. Golden, J.M.: Constructing free energies for materials with memory. Evol. Equ. Control Theory (Special Edition in Honour of Professor M. Fabrizio) 3, 447-483 (2014)

21. Golden, J.M.: Free energies for materials with memory in terms of state functionals. Meccanica 49, 2207-2235 (2014)

22. Golden, J.M.: The minimum and other free energies for non-linear materials with memory. Quart. Appl. Math. (2015, to appear)

23. Golden, J.M.: Unique characterization of materials with memory. Quart. Appl. Math. (to appear)

24. Graffi, D.: Sull'expressione analitica di alcune grandezze termodinamiche nei materiali con memoria. Rend. Semin. Mat. Univ. Padova 68, 17-29 (1982)

25. Graffi, D., Fabrizio, M.: Sulla nozione di stato materiali viscoelastici di tipo 'rate'. Atti Accad. Naz. Lincei, Rend. Lincei, Mat. Appl. 83, 201-208 (1990)

26. Graffi, D.: Ancora sull'espressione analitica dell'energia libera nei materiali con memoria. Atti R. Accad. Sci. Torino 120, 111-124 (1986)

27. Noll, W.: A new mathematical theory of simple materials. Arch. Ration. Mech. Anal. 48, 1-50 (1972) 